
allemande

49-1 | 2017

Berlin 1957-1994

\title{
Berlin: Theaterlandschaft in einer doppelten Stadt?
}

\section{Kerstin Hausbei}

\section{CpenEdition}

Journals

Édition électronique

URL : https://journals.openedition.org/allemagne/521

DOI : 10.4000/allemagne.521

ISSN : 2605-7913

Éditeur

Société d'études allemandes

Édition imprimée

Date de publication : 16 juin 2017

Pagination : $57-70$

ISSN : 0035-0974

\section{Référence électronique}

Kerstin Hausbei, „Berlin: Theaterlandschaft in einer doppelten Stadt?", Revue d'Allemagne et des pays de langue allemande [Online], 49-1 | 2017, Online erschienen am: 16 Juni 2018, abgerufen am 02 Juni 2022. URL: http://journals.openedition.org/allemagne/521; DOI: https://doi.org/10.4000/allemagne. 521 


\section{Berlin: Theaterlandschaft in einer doppelten Stadt?}

\section{- Kerstin Hausbei *}

Dass die Mathematik eine relative Kunst ist, hat meine Generation in der Bundesrepublik von Pippi Langstrumpf gelernt: „3 mal 3 macht 6, witte witte wer will's von mir lernen? Ich mach mir die Welt, witte witte wie sie mir gefällt!“ Eine antiautoritäre, ja anarchistische Mathematik, derer ich mich hier bedienen will, um einem Thema gerecht zu werden, bei dem die ,einfache‘ Mathematik schnell an ihre Grenzen stößt. „Es wird hier dröhnend, knarrend, quietschend zusammengeschraubt, was gar nicht zusammenpasst“(1), bemerkte schon Frank Castorf zu Beginn der 1990er Jahre mit Bezug auf Berlin, das ihm wie ein Fragment deutscher Geschichte erschien. Eine geteilte Stadt sollte wieder zu einer ganzen Stadt gemacht werden. Eigentlich eine einfache Rechnung: $1 / 2+1 / 2=1$. Nur dass die Hälften eben nicht mehr so nahtlos zusammengehen wollten.

Für das Theater war das vielleicht trotz allem leichter als für den Rest der Gesellschaft. So lautet zumindest das Urteil von August Everding, dem Präsidenten des Deutschen Bühnenvereins (DBV) ${ }^{(2)}$ anlässlich der Vereinigung des westdeutschen DBV mit dem ostdeutschen Bühnenbund 1990: „Wir [gemeint sind die Theaterleute] waren die einzigen, deren Taue nicht völlig gekappt und deren Wurzeln nicht radikal zerschnitten waren“(3). Im Theater hätte es demnach eine vom Rest der Gesellschaft

* Maître de conférences en études germaniques, Université Sorbonne Nouvelle-Paris 3.

1 Zitiert nach Jürgen Balitzki, Castorf, der Eisenhändler. Theater zwischen Kartoffelsalat und Stahlgewitter, Berlin, 1995, S. 232.

2 Der 1846 gegründete und 1948 neu gegründete Deutsche Bühnenverein, Bundesverband deutscher Theater ist ein eingetragener Verein der öffentlichen und privaten Träger der Theater und Kulturorchester. Er vertritt die Interessen seiner Mitglieder auf kulturpolitischem und arbeitsrechtlichem Gebiet und ist Tarifpartner auf Arbeitgeberseite. Für eine Kurzinformation zu Institutionen, und einzelnen Häusern vgl. Manfred Brauneck, Gérard Schneilin (Hg.), Theaterlexikon, Bd. 1: Begriffe und Epochen. Bühnen und Ensembles, Reinbek, 2001.

3 Festrede von August Everding, zuerst abgedruckt in: Die Deutsche Bühne, 12 (1990), hier zitiert nach Knut Lennartz, Theater, Künstler und die Politik. 150 Jahre Deutscher Bühnenverein, Berlin, 1996, S. 75. Für eine Kurzinforation zu Personen vgl. C. Bernd Sucher (Hg.), Theaterlexikon. Autoren, Regisseure, Schauspieler, Dramaturgen, Bühnenbildner, Kritiker, München, 1995. 
getrennte Mathematik gegeben, die man auf die Formel $1 \div 2=1$ bringen könnte. Auch wenn diese optimistische Einschätzung sicher zum Teil dem Gebot der Stunde geschuldet war - allein die Tatsache, dass der Bühnenbund 1990 eigens für den Zweck der Vereinigung mit dem DBV gegründet werden musste und durch Vollzug derselben im Oktober 1990 wieder aufgelöst wurde ${ }^{(4)}$, spricht hier Bände -, hatte es in der Tat in den Bühnenkünsten reale, wenn auch zahlreichen Belastungen ausgesetzte Ost-West-Beziehungen während der ganzen Zeit der deutschen Teilung gegeben. Neben politischen Entscheidungen wie Gastspiel- und Gastinszenierungspolitik, die allerdings Berlin meist gezielt aussparte, ergaben diese Kontakte sich vor allem aus den Lebensentscheidungen einzelner Künstler. Daneben gilt aber festzuhalten, dass institutionell gesehen in der geteilten Stadt eine doppelte Theaterlandschaft entstanden war. Hier müsste die Formel also lauten: $1 \div 2=1+1$. Durch die Implosion der DDR, die Wiedervereinigung und vor allem die angespannte Finanzlage der Nachwendezeit wurde diese Entwicklung dann wieder rückgängig gemacht. Auf die Formel gebracht: $1+1=1$. Ganz so mechanisch wie in unseren Rechnungen ging es natürlich nicht $\mathrm{zu}$, aber die Tendenz ist durchaus richtig, wobei Doppelung nicht als quantitative Verdoppelung, sondern als Aufbau zweier getrennter Theaterlandschaften und -systeme zu verstehen ist, wohingegen die Halbierung nicht nur die Vereinheitlichung der Theatersysteme betraf, sondern auch Theaterschließungen umfasste. Wir wollen diese Entwicklungen zunächst nachzeichnen und uns dann der Kunst und den Künstlern zuwenden und fragen, ob es, wie August Everding meint, während der Teilung „ungekappte Taue“ gegeben hat oder ob Manfred Brauneck Recht zu geben ist, wenn er angesichts der „gänzlich unterschiedlichen politischen, rechtlichen und ökonomischen Rahmenbedingungen“ zu dem Fazit kommt, diese hätten auch „die künstlerische Entwicklung des deutschen Theaters in den folgenden Jahrzehnten, bis zur Wiedervereinigung Deutschlands im Jahre 1990“ geprägt ${ }^{(5)}$.

\section{Theaterlandschaften in der geteilten Stadt oder $1 \div 2=1+1$}

Wenn man von den Theatern in öffentlicher Trägerschaft ausgeht, so steht man im geteilten Berlin vor zwei konkurrierenden Strukturen. Es gab zwei parallele Opernstrukturen (Staatsoper und Komische Oper im Osten/Deutsche Oper im Westen), zwei große Staatstheater (das Deutsche Theater mit Kammerspielen im Osten/die Staatlichen Schauspielbühnen - Schiller-Theater mit Schiller-Werkstatt und Schloßparktheater - im Westen), zwei Volksbühnen (die Volksbühne am Rosa-LuxemburgPlatz im Osten/die Freie Volksbühne im Westen) und nicht zuletzt auch zwei staatliche Schauspielschulen (die Hochschule für Schauspielkunst Ernst Busch ${ }^{(6)} \mathrm{im} \mathrm{Osten/die}$ Universität der Künste ${ }^{(7)}$ im Westen). Die Liste ließe sich noch mühelos fortsetzen:

4 Einen dem DBV vergleichbaren Verbund der Theaterträger gab es in der DDR nicht (s.u.). 1990 gegründet und aufgelöst war der Bühnenbund eine ephemere Struktur, die als Verhandlungspartner auf Ostseite fungierte, um die Theater der ehemaligen DDR in das Arbeits- und Tarifrecht der Bundesrepublik zu überführen.

5 Manfred Brauneck, Die Welt als Bühne. Geschichte des europäischen Theaters, Bd. 5: Das Europäische Theater in der zweiten Hälfte des zwanzigsten Jahrhunderts, Stuttgart, 2007, S. 207.

6 Zuvor Deutsche Schauspielschule.

7 Zunächst Hochschule für Musik und Darstellende Kunst, dann Hochschule der Künste. 
Friedrichstadtpalast und Theater des Westens für Unterhaltungskunst, Berliner Ensemble im Osten und Schaubühne am Lehniner Platz im Westen als international exponierte Bühnen. Doch da beginnt man schon, Äpfel mit Birnen zu vergleichen, denn die beiden letztgenannten Theater im Westen waren Privattheater (wie übrigens auch die Freie Volksbühne). Einen solchen Status hat es in der DDR nicht gegeben.

Die festgestellte Doppelung liegt in der Natur der Sache selbst. Theater ist eine öffentliche Kunst. Eine Kunst, die mit der Polis in Griechenland entstand und in diesem weiten Sinne eine politische Kunst ist. Eine Kunst also, die zu dem Bereich gehört, den Jacques Rancière konzeptuell als „le politique“ von der partei- und tagespolitischen Sphäre „la politique“ trennt, eine gesellschaftliche Kunst, die die verschiedensten politischen Systeme sich zunutze gemacht und auch gefürchtet haben und die so letztlich auf der Schwelle zwischen "le politique“ und „la politique“ angesiedelt ist: Repräsentation, Bildungsinstitution und kritisches Potential zugleich. Im Kalten Krieg ein Medium von entscheidender Bedeutung und Brisanz. Der Suche nach Repräsentation und der festen Verankerung des Theaters im Bildungskonzept beider deutscher Staaten und auch der vier Besatzungsmächte verdankt sich denn auch die Doppelung. Dabei kam es aber keineswegs zu einer Vermehrung der vorhandenen Spielstätten. Vielmehr konnten beide Stadthälften trotz der kriegsbedingten Zerstörung zahlreicher Theater auf gewachsene Strukturen und bestehende Häuser zurückgreifen, was freilich eine intensive Wiederaufbauarbeit, provisorische Einquartierungen und Umzüge bis in die sechziger Jahre nicht ausschloss.

Vor 1933 war Berlin eine Theatermetropole gewesen. Aufgrund der geschichtlichen Entwicklung gab - und gibt - es in Deutschland die größte Theaterdichte der Welt, ein Status, den zwischenzeitlich die „Bühnenrepublik DDR“(8) innehatte. Wie in anderen deutschen Städten geht die Struktur der öffentlichen Theater in Berlin auf die Feudalzeit zurück, in der jede Residenzstadt ihr Hoftheater besaß, das dann in der Weimarer Republik zum Staats-, Landes- oder Stadtheater umfunktioniert werden konnte $^{(9)}$. In Berlin betraf das die königlich preußischen Bühnen (die Staatsoper und das von Karl Friedrich Schinkel erbaute, im Zweiten Weltkrieg zerstörte Schauspielhaus am Gendarmenmarkt, beide im späteren Ostteil der Stadt). Gleichzeitig war im 19. und beginnenden 20. Jahrhundert vor allem in den Großstadtmetropolen Berlin und München ein dichtes Netz an bürgerlichen Privattheatern entstanden (darunter das Deutsche Theater in Berlin, das unter Max Reinhardt Weltruhm erlangte). In der Finanzkrise der zwanziger Jahre waren diese Theater zum Teil ebenfalls in öffentliche Trägerschaft überführt worden, so beispielsweise die späteren Staatstheater Westberlins: die in Charlottenburg als Bürgeroper gegründete Deutsche Oper und das Schillertheater, das 1907 in enger Zusammenarbeit mit dem Charlottenburger Magistrat als Aktiengesellschaft mit dem Auftrag gegründet worden war, die Kultur an Minderverdienende heranzutragen ${ }^{(10)}$. In Berlin gab es aber nicht nur sehr zahlreiche Thea-

8 Die Bühnenrepublik. Theater in der DDR lautet der Titel einer von Wolfgang Bergmann herausgegebenen Begleitschrift zur gleichnamigen Fernsehdokumentation, Berlin, 2003.

9 Vgl. K. Lennartz, „Theaterhistorie - Die Wurzeln der deutschen Theaterlandschaft“, in: Deutscher Bühnenverein (Hg.), Theater und Orchester in Deutschland, Köln, 2005, S. 24-35.

10 Für die Etappen des Übergangs in die öffentliche Trägerschaft vgl. den kurzen historischen Abriss aufder Internetseite des Bezirksamts Charlottenburg, http://www.berlin.de/ba-charlottenburg-wilmersdorf/ 
ter ${ }^{(11)}$, sondern hier entwickelte sich auch der größte Teil der Theater-Avantgarde, die Berlin zu einer international führenden Theatermetropole machte. In Berlin konnte man bei Max Reinhardt die erste Drehbühne der Welt bewundern (im Theater am Schiffbauerdamm 1905), hier wurden die spektakulären Piscator-Bühnen gebaut (ab 1927 im Theater am Nollendorfplatz und im Wallner-Theater). Hier wirkten Brecht, Jessner und Kortner. Hier wurde mit der Uraufführung der Stücke von Horváth und Fleißer die Grundlage für ein kritisches Volkstheater gelegt. Durch die Ausblutung der Kulturszene in der NS-Zeit war von diesem vergangenen Glanz nicht viel übrig geblieben. Wie weit die Berliner Theaterlandschaft hinter ihre einstige Größe zurückgefallen war, lässt der Vergleich ermessen, mit dem der Berliner Starkritiker aus der Weimarer Republik, Herbert Jhering, inzwischen Chefdramaturg am Deutschen Theater, seine 1947 erscheinende Abhandlung Berliner Dramaturgie eröffnete: „Keinem Pariser, keinem Moskauer, keinem Londoner, keinem New-Yorker würde es einfallen, wenn er dramaturgische Betrachtungen über die Theatersituation seiner Stadt herausgäbe, ausdrücklich zu begründen, warum er sich das Recht nähme, diese grundsätzlichen Untersuchungen nach Moskau, New York, London oder Paris zu nennen. Berlin war einst durch Jahrzehnte eine der Theaterhauptstädte der Welt, und doch muß einem Deutschen erst auseinandergesetzt werden, warum Berlin das Recht hat, [...] neue Maßstäbe zu schaffen und den Versuch einer prinzipiellen dramaturgischen Skizzierung nach sich zu benennen“(12).

Beide Stadthälften bauten also ihre Theaterlandschaft auf einem Trümmerhaufen auf, griffen dafür aber vor allem auf die Staats- und Stadtheater der Weimarer Republik zurück. Das war im Osten leichter, denn die renommiertesten Bühnen lagen im Stadtteil Mitte, beruhte aber auf einer politischen Entscheidung, die sich im Wiederaufbau wichtiger Spielstätten spiegelt. Die ehemals königlich preußische Staatsoper (dann Staatsoper der Weimarer Republik und des „Dritten Reiches“) wurde (1955 wieder aufgebaut) zur Staatsoper der DDR. Schon 1947 war die Komische Oper im wieder aufgebauten Metropoltheater eröffnet worden. Das ehemalige Staatstheater, das Schauspielhaus am Gendarmenmarkt, wurde nicht wieder aufgebaut, aber mit dem Deutschen Theater gab es eine prestigereiche Alternative. Die (1954 wieder aufgebaute) Volksbühne, das historische Haus der Besucherorganisation Freie Volksbühne, an dem Reinhardt und Piscator gewirkt hatten, unter dem Nationalsozialismus durch Enteignung verstaatlicht, wurde zum Ostberliner Stadttheater. Neugegründet wurde das Maxim Gorki Theater, das man mit dem Auftrag der Pflege sowjetischer Dramatik in der ehemaligen Singakademie einquartierte. Als 1949 Bertolt Brecht und Helene Weigel die Möglichkeit gegeben wurde, das Berliner Ensemble zu gründen, musste dieses bis 1954 im Deutschen Theater mit dem dortigen Ensemble unter der

ueber-den-bezirk/kultur-und-wissenschaft/buehnen/artikel.180256.php [zuletzt eingesehen am 21.2.2017]. Zur Geschichte der Theatergebäude vgl. auch Harald ZiELSKE, „Thalia in urbaner Enge. Theaterstandorte und Theaterbau in Berlin 1890-1990“, in: Erika Fischer-Lichte, Doris KollesCH (Hg.), Berliner Theater im 20. Jahrhundert, Berlin, 1998, S. 53-76.

11 Die Berlin Tourismus \& Kongress GmbH wirbt heute wieder damit, dass Berlin über 150 Spielstätten verfügt: http://www.visitberlin.de/sites/default/files/berlin_in_zahlen_wussten_sie_schon_0.pdf (zuletzt eingesehen am 21.2.2017).

Herbert Jhering, Berliner Dramaturgie, Berlin, 1947, S. 5. 
Intendanz von Wolfgang Langhoff kohabitieren, bevor es sich, nachdem das Volksbühnenensemble wieder ins eigene Haus umgezogen war, als weiteres Staatstheater der DDR im Theater am Schiffbauerdamm (auch dies ein Wirkungsort von Brahm und Reinhardt und Spielstätte des kritischen Volkstheaters) etablierte. 1984 wurde schließlich mit dem neuen Friedrichstadtpalast der letzte Prachtbau der DDR eingeweiht: mit $3.000 \mathrm{~m}^{2}$ bespielbarer Fläche ist er bis heute die größte Theaterbühne der Welt. Alle diese Theater liegen in Fußnähe zueinander. Privattheater existierten in der DDR bis in die 1980er Jahre nicht. Allerdings gab es viele Amateurtheatergruppen, die von professionellen Regisseuren geleitet wurden.

In Westberlin hatte man es ungleich schwerer. Nicht nur musste man auf ehemals private Spielstätten in der Peripherie des historischen Theaterzentrums zurückgreifen, sondern die Spielstätte der Deutschen Oper war auch durch Kriegsschäden vollständig zerstört, was dazu führte, dass das Ensemble zunächst ins Theater des Westens ausweichen musste, was aber keine dauerhafte Lösung war. Der nach den Plänen von Fritz Bornemann erstellte Neubau in Charlottenburg (in unmittelbarer Nähe zum ursprünglichen Standort) stand erst 1961 zur Verfügung. Bei der Gründung der Staatlichen Schauspielbühnen entschied man sich 1951 für das Schillertheater. Damit war in Westberlin der Schwerpunkt der öffentlichen Theater nach Charlottenburg gelegt worden $^{(13)}$, eine Entscheidung gegen Kreuzberg, wo das Hebbel-Theater stand, auf das die Besatzungsmächte, vor allem unter der Intendanz des von ihnen berufenen und 1948 verstorbenen Karl Heinz Martin, für die kulturelle reeducation gesetzt hatten. Neben den Theatern in öffentlicher Trägerschaft gab es in Westberlin ein dichtes Netz an Privattheatern, von denen viele der in der direkten Nachkriegszeit (vor allem mit amerikanischen Lizenzen) neu gegründeten allerdings die durch die Währungsreform entstandene Krise nicht überstanden ${ }^{(14)}$. Die Freie Volksbühne, die 1947 als Zuschauerorganisation in beiden Stadtteilen getrennt wiedergegründet worden war, blieb im Westen ihrem historischen Status als eingetragener Verein treu ${ }^{(15)}$, musste aber - da die historische Spielstätte im Ostteil der Stadt lag - zunächst das Theater am Kurfürstendamm mieten, bis 1963 ein eigenes Haus im selben Viertel gekauft werden konnte $^{(16)}$. Es war so letztlich ein Ost-West-Gefälle in der Bedeutung der Häuser vorprogrammiert, das sich durch den verschiedenen Status der Stadthälften im jeweiligen Staat noch verschärfte: während Ostberlin als Hauptstadt zur Theatermetropole der DDR wurde, entstand (auch aufgrund der dezentralen Lage und der Teilung von Berlin) in der Bundesrepublik eine dezentrale Struktur, in der Westberlin sich als Theaterstadt kaum neben den alten und neuen Zentren wie München, Hamburg, Düsseldorf,

13 Das Schloßparktheater als kleines Haus der Staatlichen Spielstätten war allerdings in Steglitz angesiedelt.

14 Vgl. Peter Jammerthal, Henning Rischbieter, „Hektischer Betrieb nach Kriegsende - TheaterFrontbildungen im Kalten Krieg 1945-1948“, in: Henning Rischbieter (Hg.), Durch den Eisernen Vorhang. Theater im geteilten Deutschland 1945 bis 1990, Berlin, 1999, S. 20, und mit leicht abweichenden Zahlen M. Brauneck, Welt als Bühne (Anm. 5), S. 206.

15 Die Freie Volksbühne war 1890 im Zuge der Volksbühnenbewegung in ganz Europa in Berlin als Verein gegründet worden, um die Zensur zu umgehen, die auf geschlossene Aufführungen keinen Einfluss nehmen konnte. Sie verhalf dem Naturalismus zum Durchbruch.

16 Heute sind dort die Berliner Festspiele untergebracht. 
Bochum, Stuttgart, Frankfurt, Bremen und Darmstadt behaupten konnte. Seit den 1960er Jahren entwickelte sich in Westberlin wie in der gesamten Bundesrepublik eine umfangreiche freie Theaterszene, deren prominentester Vertreter sicher die 1962 von ehemaligen Studierenden der Freien Universität gegründete und zunächst im Theater am Halleschen Ufer untergebrachte Schaubühne war. Da Peter Stein und sein Ensemble die internationale Aufmerksamkeit auf sich zogen und die Schaubühne zum „Werbeschlager“ (Friedrich Luft ${ }^{(17)}$ ) für Westberlin machten, erlangten sie eine Verhandlungsbasis zur Erhöhung der zunächst bescheidenen Subvention von 1,8 Millionen DM und vor allem für den Umzug in die größere Spielstätte am Lehniner Platz $(1981)^{(18)}$. Die Schaubühne erhielt dadurch einen Status, der sich letztlich nicht sehr von dem eines Staatstheaters unterschied. Das Haus am Halleschen Ufer blieb in den Händen der freien Theaterszene. Es wurde zunächst zur Spielstätte der Theatermanufaktur, einer 1970 gegründeten Gruppe, und nach der Wende (1992) zur zentralen Spielstätte der freien Gruppen Berlins ${ }^{(19)}$. In der Freien Theaterszene kam es auch zu einer publikumsorientierten Ausdifferenzierung. Das von Volker Ludwig 1966 als Kindertheater gegründete GRIPS-Theater ist das prominenteste Beispiel und spielt bis heute eine bedeutende Rolle in der Theaterlandschaft Berlins.

\section{Theatersysteme in der geteilten Stadt oder $1 \div 2=1+1$}

Diese Theaterlandschaften in Ost und West waren während der Teilung an getrennte Theatersysteme rückgebunden, in denen die Intendanten, Regisseure und Schauspieler sehr verschiedene Arbeitsbedingungen hatten. In beiden Staaten war das Theater dezentral organisiert, hing also von lokalen oder regionalen politischen Instanzen ab. Diese spielten aber eine nicht vergleichbare Rolle in beiden Stadthälften. Im Osten bestand eine direkte Abhängigkeit der Theater von der Politik. Es wurde Einfluss genommen auf die Spielplangestaltung, die Programmhefte sowie berufliche Belange wie die Künstlervermittlung und die ,Kaderbildung' an den Theatern. Die Tarife wurden gesetzlich festgelegt. Ab 1978 erhielten die Bühnenkünstler der DDR unbefristete Arbeitsverträge und waren praktisch unkündbar. Auch der als Volksbühne 1947 wiedergegründete Zuschauerverein (ehemals und im Westen wieder Freie Volksbühne) wurde wie alle Zuschauerorganisationen ins System überführt und war nun Teil des FDGB. Für die Ostberliner Staatstheater als Hauptstadttheater war seit 1954 direkt das Kulturministerium unter Johannes R. Becher zuständig, sowie die 1958 gegründete „Kommission für Fragen der Kultur“ beim Politbüro der SED unter der Leitung von Alfred Kurella. Die eigentlichen Entscheidungen fielen denn auch in der Partei. Neben dem bekannten ,Kahlschlag-Plenum' von 1965 befassten sich auch die Tagungen des Zentralkomitees der SED mit Theater. Es wurde dort über Stückeund Inszenierungsverbote befunden, aber auch Personalpolitik betrieben. 1963 wurde hier beispielsweise die als Rücktritt verschleierte Absetzung von Wolfgang Langhoff

17 Zitiert nach M. Brauneck, Welt als Bühne (wie Anm. 5), S. 305.

18 Vgl. Monika Sandhack, „Schaubühne am Lehniner Platz“, in: Braunek/Schneilin (Hg.), Theaterlexikon 1 (Anm. 2), S. 878f.

19 Seit 2003 ist das Theater am Halleschen Ufer mit dem Hebbel-Theater und dem Theater am Ufer als Hebbel am Ufer (HAU 1, 2, 3) zusammengeschlossen. 
als Intendant des Deutschen Theaters beschlossen. Die politischen Übergriffe betrafen auch rein künstlerische Fragen. Inhaltlich und ästhetisch war der Rahmen des, sozialistischen Realismus' verbindlich - das hieß eine ideologische Normierung der Spielpläne durch Festlegung auf „positive Helden des sozialistischen Aufbaus“ und auch eine ästhetische Normierung im Sinne einer Inszenierungs- und Schauspieltradition des einfühlenden Spiels, wie sie vom russischen Regisseur Konstantin S. Stanislavskij begründet worden war ${ }^{(20)}$ und wie sie beispielsweise bei der Stanislawski-Konferenz 1953 in Ostberlin verkündet wurde. Festgelegt wurde auch die Orientierung am Vorbildcharakter des bürgerlich-humanistischen Kulturerbes und der bürgerlichen Freiheitsbewegung des 19. Jahrhunderts. Alles andere - vor allem die avantgardistischen Ansätze der Weimarer Republik - wurden als Formalismus diskreditiert. Es setzte sich so die Frontstellung der 1930er Jahre ungebrochen fort. Die Etablierung von Brecht in Ostberlin führte allerdings zu einem Spannungsfeld, das sich zunächst im Haus des Deutschen Theaters in extremer Konzentration entwickelte, da dort bis 1954 Brecht als Vertreter des epischen Schauspielstils und Wolfgang Langhoff als Hauptvertreter der Stanislavskij-Ästhetik auf engstem Raum nebeneinander arbeiten mussten.

In Westberlin war der Kultursenator zuständig. Seine Rolle war begrenzt. Ein Theatergesetz gibt es in der Bundesrepublik nicht. Tarifverträge wurden - und werden für die ganze Bundesrepublik zwischen der Vertretung der Theaterleitungen, also dem DBV, und der Genossenschaft Deutscher Bühnenangehöriger ausgehandelt ${ }^{(21)}$. Die zentrale Figur im Theater der Bundesrepublik ist seit 1949 der Intendant. Er hat das alleinige Recht zu engagieren und den Spielplan zu gestalten. Er ist dementsprechend souverän gegenüber der Politik. Die politische Sphäre spielt als Theaterträger eine vor allem finanzielle Rolle, hat aber durch die Besetzung von Intendantenstellen und die Zuteilung von Subventionen trotz allem eine nicht unbeträchtliche Möglichkeit der indirekten Einflussnahme. In Bezug auf die Personalpolitik war von entscheidender Bedeutung, dass die westlichen Alliierten und die Stadt Westberlin (wie auch die übrige Bundesrepublik) keine Politik der Rückkehr von exilierten Bühnenkünstlern betrieben. Die Intendanten der 1950er Jahre wurden aus den Theaterschaffenden der NS-Zeit rekrutiert. Die personale Kontinuität wirkte sich auf die Spielplangestaltung, aber auch auf den Regiestil aus, der noch stark von der NS-Ästhetik geprägt blieb. Fritz Kortner und Berthold Viertel nannten das den „Reichskanzleistil“ (22). Ein Anknüpfen an die politisch orientierte Modernität der Weimarer Republik war dadurch unmöglich. Es gab auf der einen Seite Vertreter der Werktreue, auf der anderen Seite ein Modernitätskonzept, das mit Mitteln der Abstraktion und der Enthistorisierung die Grundfragen der Existenz zu erschließen versuchte. An der Freien Volksbühne stand

20 Es ist ein Paradox des Kalten Krieges, dass sich diese Schauspieltradition nach der Tournee des Moskauer Künstlertheaters von 1927 auch in den USA etabliert hatte und mit dem 1947 gegründeten Actors Studio maßgeblichen Einfluss auf das amerikanische Theater und Kino erhielt und bis heute behält.

21 Vgl. (aus Arbeitgebersicht): Rolf Bolwin, „Die rechtliche Struktur - Tarifverträge und Arbeitsverträge“, in: Deutscher Bühnenverein (Hg.), Theater und Orchester in Deutschland (Anm. 9), S. 124-139.

22 Vgl. vor allem Berthold Viertel, „Der Reichskanzleistil“, in: Ders., Die Überwindung des Übermenschen. Exilschriften (Studienausgabe, Bd. 1), hg. von Konstantin KaISER und Peter Roessler, Wien, 1989, S. 275-277. 
Oscar Fritz Schuh für ein für die 1950er Jahre typisches Konzept des Theaters als ,geistigen Raums ${ }^{(23)}$, eines Ortes also, an dem „Fragen der menschlichen Existenz jenseits von Geschichte und Politik“verhandelt werden ${ }^{(24)}$.

In dieser Atmosphäre der Entpolitisierung hatten es die Rückkehrer entsprechend schwer. Fritz Kortners Hoffnung auf die Intendanz des Schiller-Theaters zerschlug sich nach dem Skandal um seine Don Carlos-Inszenierung 1950, in der er Don Carlos als unheldischen Verlierer gegen das System interpretiert hatte ${ }^{(25)}$. Er pendelte weiter ohne eigenes Theater zwischen Berlin und München. Auch Erwin Piscator schaffte es zunächst nicht, wieder Fuß zu fassen. Den USA zu kommunistisch, der UdSSR zu trotzkistisch, der DDR zu avantgardistisch, der Bundesrepublik zu politisch saß er zwischen allen Stühlen, arbeitete zunächst zehn Jahre als Gastregisseur an westdeutschen Bühnen und musste Kompromisse eingehen. Er versuchte in dieser Zeit, Friedrich Schiller wieder von der nationalen Überhöhung zu befreien und als Revolutionär des 18. Jahrhunderts sichtbar zu machen, wobei er allerdings nicht mehr mit einer direkten Aktualisierung wie in den 1920er Jahren arbeitete. Mit Stücken wie Gas von Georg Kaiser nahm er vorsichtig Stellung zu tagespolitischen Themen wie dem der atomaren Bewaffnung. Seine Hoffnung auf eine parteipolitische Anbindung an die SPD zerschlug sich 1958 an der gleichen Frage. Als er 1962 mit der Freien Volksbühne endlich ein eigenes Haus übertragen bekam (bezeichnenderweise kein öffentliches Theater), positionierte er das Haus in der Eröffnungsrede der neuen Spielstätte (1963) jenseits der Parteipolitik ${ }^{(26)}$. Es ging ihm nun darum, ein Forum für die kritische Befragung der Gesellschaft zu schaffen. Piscator setzte dabei vor allem auf die neue Autorengeneration - Rolf Hochhuth, Heinar Kipphardt, Peter Weiss - und wurde zum Geburtshelfer des neuen Dokumentartheaters nicht nur dadurch, dass er die Stücke zur Uraufführung brachte, sondern auch dadurch, dass er sich für ihre Verbreitung - beispielsweise durch Ring-Uraufführungen - einsetzte. In seinen Inszenierungen legte er den Akzent auf den Umgang mit der NS-Vergangenheit und die Frage der Verantwortung des Wissenschaftlers und entschärfte diejenigen Aspekte der Stücke, die im Kontext des Kalten Krieges (gerade in Berlin) durch ihre Kapitalismuskritik tagespolitisch aufgeladen werden konnten ${ }^{(27)}$. Seine Intendanz an der Freien Volksbühne rückte Westberlin ins Zentrum des internationalen Interesses und wurde zum Anstoß einer Politisierung des Theaters im Sinne eines modernen Denktheaters.

Neben der Intendantenbesetzung standen in Westberlin aber auch Versuche direkter Eingriffe in den Entscheidungsbereich der Intendanten. Dabei ging es zunächst um die Konkurrenz mit Ostberlin. In der dezentralen Struktur der Bundesrepublik war für die Karriere eines Bühnenkünstlers ein Engagement nach Westberlin kein Aufstieg,

23 Vgl. Oscar Fritz Schuн, Die Bühne als geistiger Raum, Bremen, 1963.

24 M. Brauneck, Welt als Bühne (Anm. 5), S. 218.

25 Vgl. Henning Rischbieter, „Im Gefängnis der Tyrannei. Kortners Don Carlos-Inszenierung, WestBerlin 1950“, in: Fischer-Lichte/Kollesch (Hg.), Berliner Theater im 20. Jahrhundert (Anm. 10), S. 171-184.

26 Abgedruckt in: Erwin Piscator, Eine Arbeitsbiographie, Bd. 2, hg. von Kurt Boeser u. Renata VAtKová, Berlin, 1986.

27 Vgl. Klaus Wannemacher, Erwin Piscators Theater gegen das Schweigen. Politisches Theater zwischen den Fronten des Kalten Krieges (1951-1966), Tübingen, 2004. 
wohingegen die westdeutschen Künstler, solange das noch möglich war, gerne ihren Gastspielurlaub dazu nutzten, in der Staatsoper, der Komischen Oper oder dem Deutschen Theater aufzutreten oder auch gleichzeitig in West- und Ostberlin unter Vertrag standen. Im Kontext des Kalten Krieges hatte das zur Konsequenz, dass der Westberliner Senat, aber auch das Bundesministerium für gesamtdeutsche Fragen und der Deutsche Bühnenverein seit 1951 darauf drangen, „die an den Westberliner Bühnen beschäftigten Künstler [sollten] nur noch hier und nicht zugleich auch im Osten auftreten “(28). Auch wurden die westdeutschen Theater dazu aufgerufen, „Gastspiele geschlossener Ensembles aus Ostberlin oder der Ostzone [genannt werden das Berliner Ensemble und das Deutsche Theater] grundsätzlich abzulehnen“(29). Begründet wurde das wie folgt: "Jedes Ensemble aus dem Osten, das nach Westdeutschland geht, dient dem Zweck, dort eine Kulturfassade aufzurichten, hinter der sich der Kommunismus zu verbergen sucht, auch dann, wenn die Mitglieder dieses Ensembles ihn persönlich ablehnen und keine derartigen Absichten verfolgen “(30). Damit wurde von westlicher Seite eine Spaltung der Theaterlandschaft in Berlin betrieben, die zuvor noch nicht bestanden hatte. Aus der Analyse der Spielpläne der Endvierzigerjahre ergibt sich, dass die Regisseure sich zunächst noch frei zwischen den Zonen hin- und herbewegt hatten ${ }^{(31)}$. Bis zum Mauerbau lebten auch etwa 1.000 an den Ostberliner Theatern tätige Musiker, Sänger und Schauspieler im Westteil der Stadt. Diese vom Westberliner Arbeitsamt als „Auspendler" bezeichneten Künstler wurden nach dem Mauerbau von der DDR gezwungen, sich innerhalb eines Monats zu entscheiden, ob sie in den Ostteil der Stadt umziehen oder ihr Engagement verlieren wollten. Viele entschieden sich für den Westen, was in Westberlin, wo es ohnehin viele erwerbslose Bühnenkünstler gab, zu einem kaum lösbaren Problem führte, das noch dadurch verschärft wurde, dass die Angestellten der Ostbühnen in der Bundesrepublik nicht arbeitslosenversichert waren und dadurch keinerlei Ansprüche auf Leistungen geltend machen konnten ${ }^{(32)}$. Nur wenige Prominente Ostberliner Künstler konnten auch nach dem Mauerbau ihren Wohnsitz im Westen behalten. Zu ihnen zählten Walter Felsenstein, der Intendant der Komischen Oper, Erich Engel, Regisseur am Berliner Ensemble, Gerhart Bienert, Schauspieler am Deutschen Theater und Hans Nocker, Sänger in Felsensteins Ensemble ${ }^{(33)}$. Im Westen

28 Nachrichtendienst des Deutschen Bühnenvereins, 4 (1951), abgedruckt in K. LenNARTz, Theater, Künstler und die Politik (Anm. 3), S. 297. Die zitierte Passage betrifft ein vom Bundesminister für gesamtdeutsche Fragen dem DBV „mit der Bitte, sich nach diesen Grundsätzen zu richten“, übermitteltes Gutachten. Bei Lennartz ebenfalls abgedruckt findet sich ein Bericht aus der SPD-nahen Westberliner Zeitung Der Telegraf von 1955, in dem über einige spektakuläre Fälle berichtet wird, in denen die Westberliner Behörden gleichzeitige Engagements in West- und Ostberlin als vertragswidrig zurückwiesen. Vgl. ebd., S. 301.

29 Ebd.

30 Ebd., S. $297 \mathrm{f}$

31 Vgl. dazu die Zeittafel in Ursula Heukenkamp (Hg.), Unterm Notdach. Nachkriegsliteratur in Berlin 1945-1949, Berlin, 1996, S. 453-528.

32 Vgl. den Brief des Deutschen Städtetags „Betr.: Maßnahme zur Unterbringung Berliner Künstler“ vom 11.10.1961, abgedruckt im Quellenteil „Zeitzeugnise zur Geschichte des deutschen Theaters und des deutschen Bühnenvereins“, in: K. Lennartz, Theater, Künstler und die Politik (Anm. 3), S. $310 \mathrm{f}$.

33 Vgl. C. B. Sucher, Theaterlexikon (Anm. 3), sowie K. Lennartz, Theater, Künstler und die Politik (Anm. 3), vor allem Kapitel VIII „Ost-West-Beziehungen“. 
antwortete man auf diese Situation - wie schon 1953 und 1956 - mit dem Aufruf zu einem Brecht-Boykott, der in Westberlin länger durchgehalten wurde als im Rest der Bundesrepublik. Allerdings reagierten 68 Intendanten der Bundesrepublik und Westberlins diesmal mit einer Erklärung, in der sie „vor jeder tendenziösen Beeinflussung der Spielpläne durch Gruppen außerhalb des Theaters nachdrücklich“ warnten $^{(34)}$.

$\mathrm{Zu}$ einer ähnlichen Auseinandersetzung kam es noch einmal 1970 im Zusammenhang der Mitbestimmungsexperimente in Berliner Theatern. Nach dem Tod Piscators war zunächst die Freie Volksbühne in die Politisierung der End-1960er Jahre hineingerissen worden. Unter der Intendanz von Hansjörg Uzerath unterbrachen die Schauspieler eine Horváth-Inszenierung, um einen Resolutionsentwurf gegen die Notstandsgesetze zu verlesen und zu diskutieren. Die Freie Volksbühne hatte sich so vom Politischen zur Politik entwickelt - von "le politique“ zu "la politique“. Diese Entwicklung mündete in der Spielzeit 1969/70 erstmals in die Entstehung eines eigenen Ensembles, das mit der Einführung gleicher Gagen für alle und einer kollektiven Leitung Akzente setzte, die dann an der Schaubühne weitergeführt wurden und das Theater zu einem Experimentierfeld für gesellschaftliche Entwicklungen machen sollten. Für kurze Zeit kam es so zu der Forderung der Aufhebung zwischen Kunst und Leben, einer Konzeption der Kultur für alle, in der Autorschaft und Werkcharakter infrage gestellt wurden. 1970 erhob die CDU-Fraktion des Berliner Abgeordnetenhauses gegen Peter Steins Ensemble Anklage mit dem Vorwurf, dieses habe Subventionsgelder zweckentfremdet, um politische Agitation für den Kommunismus zu betreiben. Zu einer Großoffensive des DBV kam es dann nicht mehr, da die gesperrte Subvention nach Entkräftung der Vorwürfe ausbezahlt wurde ${ }^{(35)}$. Neben diesen repressiven politischen Eingriffen wurde vom Westberliner Senat auch eine voluntaristische Politik betrieben, um Westberlin als Theaterstadt aufzuwerten. Das begann 1951 mit der Gründung der Berliner Festwochen (im Rahmen der Berliner Festspiele), die, zunächst von den Westalliierten finanziert, als Vitrine internationaler westlicher Bühnenkunst konzipiert waren. Auf Vorschlag des Berliner Bürgermeisters Suhr wurde 1956 vom Bundestagsausschuss Film, Presse, Funk ein Umtauschkurs von 1:1 für den Besuch Westberliner Kulturstätten eingeführt, um das Ostberliner Publikum anzulocken. Der Magistrat Ostberlins konterte 1958 mit der Schaffung eines eigenen Festivals, den Berliner Festtagen. 1964 wurde dann in Westberlin das Berliner Theatertreffen gegründet, ein im deutschen Sprachraum führendes Festival, zu dem eine Kritikerjury eine Selektion von herausragenden Produktionen der Vorsaison aus allen deutschsprachigen Ländern einlud. Auch Produktionen aus Ostberlin wurden immer wieder eingeladen, was aber von der DDR boykottiert wurde. Ihren Höhepunkt erreichte diese Politik 1970 mit der politisch umstrittenen Anwerbung von Peter Stein an die Schaubühne, die Westberlin in den Blickpunkt internationaler Aufmerksamkeit brachte. Stein kam nur deshalb nach Berlin, weil er als Regisseur 1969 nicht in München bleiben konnte, nachdem er die Parteilichkeit des Vietnam-Diskurses von Peter Weiss so weit hatte treiben wollen, eine Sammelaktion im Publikum für die Bewaffnung des Vietkong zu starten. Gestützt durch das Berliner Theatertreffen 
wurde dann mit dem Regietheater ein neues Konzept von Autorschaft geschaffen, das sich diesmal auf den Regisseur bezog. Dabei stand aber nicht mehr die Politisierung im Mittelpunkt. So irritierte auch Peter Stein den politisierten Erwartungshorizont, indem er zwar analytisch-kritisch inszenierte, dabei aber eher auf psychologische Fallstudien setzte denn auf sozialkritische Analyse.

\section{Theatersystem und -landschaft in der wiedervereinigten Stadt oder $1+1=1$}

Nach der Wende kam dann das Nachspiel der Doppelung. Das Theatersystem der DDR wurde schlicht in das Theatersystem der Bundesrepublik überführt, eine beängstigende Situation für die Bühnenkünstler der ehemaligen DDR, die nun den arbeitsund tarifrechtlichen Verhältnissen der Bundesrepublik unterstellt wurden und damit ihre unbefristeten Arbeitsverträge verloren. In der angespannten Finanzlage der Nachwendezeit - dem Theaterkritiker Urs Jenny zufolge ging es um eine Kürzung des Berliner Kulturetats um 60 Millionen D-Mark ${ }^{(36)}$ - wurde auch die Doppelung der Theaterlandschaften rückgängig gemacht, ein schmerzhafter Prozess, dem zunächst 1991 die Freie Volksbühne und dann 1993 die Staatlichen Schauspielbühnen Berlin (Schiller-Theater, Schiller-Werkstatt und Schloßparktheater) zum Opfer fielen, was dem damaligen Kultur-Senator Ulrich Roloff-Momin den Beinamen „Schiller-Killer“ einbrachte. Das Budget der Volksbühne und das des Deutschen Theaters wurden hingegen (geringfügig) aufgestockt. Das ehemalige DDR-Staatstheater Berliner Ensemble wurde in ein privatrechtliches Unternehmen umgewandelt, dem nun ein Direktorium vorstand, dem neben Heiner Müller noch Peter Zadek, Peter Palitzsch, Fritz Marquart und Matthias Langhoff angehörten, mit Ausnahme von Peter Zadek eine Mischung aus Zurückgekehrten und Dagebliebenen aus DDR-Zeiten. Wenn Matthias Lilienthal als Chefdramaturg der Volksbühne in den 1990er Jahren eine „Entwertung von allem, was in der DDR bestand (mit dem bloßen Argument, dass es zur DDR gehörte)“(37) diagnostizierte, so traf das also auf die Theaterlandschaft gerade nicht zu.

Die Entscheidung gegen die Westtheater kann verschieden begründet werden. Einerseits ergab sie sich recht logisch aus dem historischen Status der Häuser vor der Teilung, dem Ost-West-Gefälle während der Teilung und auch aus ihrer Lage in der Stadt. Nach der Wiedervereinigung orientierte sich das Westberliner Publikum zurück in das alte Theaterzentrum der Stadt und frequentierte vor allem wieder das Deutsche Theater und das Berliner Ensemble. Antje Dietzke sieht das als Teil einer „allgemeinen räumlichen Verschiebung im Kulturbereich der Stadt nach der Maueröffnung [...] in die historische Stadtmitte, die sich innerhalb kurzer Zeit wieder zum kulturellen Zentrum der Stadt entwickelte“(38). Andererseits spielte der im Einigungsvertrag festgelegte Substanzschutz für die DDR-Theater eine Rolle. Der Bund sorgte

36 Vgl. Urs Jenny, „Notstand ist jetzt“, Der Spiegel, 26 (1993), S. 206-208.

37 Matthias Lilienthal, „Die Chance des Scheiterns der Betonarbeiterbrigade“, in: Hans-Dieter SCHÜTt, Castorfs Volksbühne. Schöne Bilder vom häßlichen Leben, Berlin, 1999, S. 37.

38 Antje Dietzke, Ambivalenzen des Übergangs. Die Volksbühne am Rosa-Luxemburg-Platz in Berlin in den neunziger Jahren, Göttingen, 2014, S. 176. Ausführliche Darstellung der Krise und Umstrukturierung der Berliner Theaterlandschaft nach der Wende im Kapitel „Die Berliner Theaterlandschaft der neunziger Jahre“. 
für eine Übergangsfinanzierung, die zwar die Finanzkrise des Landes Berlin, das zum neuen Träger der Theater geworden war, nicht ausglich, aber doch die Krise für die Ostberliner Theater abschwächte.

\section{Ungekappte Taue? Oder doch unüberwindliche Unterschiede?}

Wendet man sich der künstlerischen Entwicklung in beiden Stadthälften zu, so gilt zunächst festzuhalten, dass in beiden Stadthälften - wie auch in beiden Staaten - die kritische Auseinandersetzung mit der je eigenen Gesellschaft im Vordergrund stand. Wenn Manfred Brauneck rückblickend feststellt, dass das historisch wichtigste Ereignis der deutschen Nachkriegsgeschichte, die Teilung der Nation, an den bundesdeutschen Bühnen beinahe spurlos vorübergegangen ist ${ }^{(39)}$, so ließe sich Ähnliches vielleicht auch für die DDR und Ostberlin sagen. Denn dort ging es vom Lohndrücker Heiner Müllers bis hin zur Übergangsgesellschaft Volker Brauns in verschiedener Weise um die nicht eingelösten Hoffnungen in der sozialistischen Gesellschaft. Dabei lassen sich verschiedene Phasen in den Reaktionen des Regimes auf Kritik feststellen, die wiederum zu verschiedenen Strategien auf Seiten der Künstler führten. Nach dem Verbot der konstruktiven Kritik der Dramatik von Müller und Hacks, die im Zuge des Bitterfelder Wegs entstanden war, wichen die Regisseure zunächst auf Klassikerinszenierungen aus, die sie allerdings oppositionell inszenierten. Auch das war gefährlich. Beispielsweise musste infolge der Faust-Inszenierung von Adolf Dresen 1968 Wolfgang Heinz die Intendanz des Deutschen Theaters aufgeben. Trotzdem wurde dieser Weg - auch am Deutschen Theater - weiterverfolgt (etwa durch Alexander Lang). In den 1970er Jahren kamen dann auch Inszenierungen von unliebsamen Stücken (beispielsweise des Prinzen von Homburg) hinzu. Andere Stücke - wie Goethes Iphigenie - wurden radikal uminterpretiert. In der Dramatik war das eine Zeit, in der - etwa bei Heiner Müller - die Auseinandersetzung mit der in der DDR verdrängten (Kontinuität der) NS-Vergangenheit in den Blick geriet. Während die entsprechenden Stücke aber nicht an Ostbühnen aufgeführt werden konnten, kamen nun zeitversetzt die in den 1960er Jahren verbotenen Stücke auf die Bühne. Insgesamt bildete sich an den Ostberliner Bühnen wie in der übrigen DDR eine subtil verbergende politische Kommunikation mit dem Publikum heraus, die dem Theater den Status eines ,Medienersatzes ' einbrachte. Auch wenn für das Publikum durch die vom RIAS übertragenen Theaterkritiken von Friedrich Luft und durch die Besprechungen von Ostberliner Produktionen in der Westpresse die Möglichkeit der Information über die jeweils andere Theaterlandschaft gegeben war, waren auf diese Weise doch vollkommen unterschiedliche Resonanzräume entstanden, die es auch schwer machten, Stücke, die in dem einen Kontext entstanden waren, in den anderen zu überführen, worauf Heiner Müller mit Bezug auf Der Auftrag und den Viet Nam Diskurs von Peter Weiss verweist ${ }^{(40)}$.

Was die Künstler angeht, so ist allerdings von Mobilität und Informiertheit auszugehen. Anders als im Westen war man im Osten bemüht gewesen, Bühnenkünstler aus dem Exil zurückzuholen. Die sowjetische Besatzungszone wurde so zunächst zum Sammelbecken für linksbürgerliche und sozialistische Kreise, die die Ideen

39 Vgl. M. Brauneck, Welt als Bühne (Anm. 5), S. 350.

40 Vgl. Heiner Müller, Krieg ohne Schlacht, Köln, 1992, S. 298f. bzw. 222f. 
der Volksfront aus den 1930er Jahren wieder aktivieren wollten. Schon 1947 erfolgte allerdings die Umorientierung der Spielpläne, die sich zunächst auf eine antifaschistische, demokratische Neuordnung konzentriert hatten, hin zu einer klaren Propagandalinie im Kalten Krieg. Viele Bühnenkünstler und Autoren kamen trotzdem in der Anfangszeit der DDR aus dem Westen (einschließlich der Schweiz und Österreich) nach Ostberlin. Neben Brecht und Helene Weigel, die ein wichtiger Anziehungspunkt für viele andere wurden, traf das beispielsweise auch auf Wolfgang Langhoff und Wolfgang Heinz (Intendanten am DT) zu, ebenso auf den Chefdramaturgen am DT, Herbert Jhering, sowie auf dessen Nachfolger, den Autor Heinar Kipphardt. Der Intendant der Volksbühne, Fritz Wisten, war vom NS-Regime verfolgt worden. Der Autor Peter Hacks kam noch 1955 als Dramaturg ans Berliner Ensemble. Viele kamen und blieben wegen Brecht. In Krieg ohne Schlacht erklärt Heiner Müller: „Brecht war die Legitimation, warum man für die DDR sein konnte. [...] Weil Brecht da war, mußte man dableiben. [...] Brecht war das Beispiel, daß man Kommunist und Künstler sein konnte - ohne das oder mit dem System, gegen das System oder trotz des Systems “(41). Letztlich wiederholte sich aber immer wieder das Brecht-Paradox. Die aktive Mitarbeit von überzeugten Sozialisten am Aufbau der sozialistischen Gesellschaft wurde von der Partei behindert oder direkt unterbunden. Daran scheiterten letztlich alle, auch dann noch, als Brecht schon zum Klassiker erhoben war. Deshalb verlief der Weg der Künstler später umgekehrt. In mehreren Schüben gingen Künstler von Ostberlin in die Bundesrepublik: Peter Palitzsch, Manfred Karge und Matthias Langhoff, Benno Besson, Jürgen Gosch, Einar Schleef, Alexander Lang, Adolf Dresen sowie die Autoren Kipphardt und Thomas Brasch, um nur einige zu nennen. Sie wurden zu zentralen und geschätzten Bestandteilen der westdeutschen und europäischen Theaterlandschaft und $\mathrm{zu}$ wesentlichen Vermittlern der Theatertheorie und -praxis von Brecht. Die Wertschätzung des Osttheaters lässt sich auch an den zahlreichen Einladungen von Produktionen zum Berliner Theatertreffen ablesen, die allerdings bis 1989 von der DDR abgewiesen wurden ${ }^{(42)}$. Die DDR reagierte auf diese Entwicklungen indem sie Ostregisseuren die Möglichkeit einräumte, im Westen zu inszenieren, vielleicht in der Hoffnung, manche würden Rückweg nicht mehr antreten. Der ästhetische Austausch war vielfältig.

Auch wenn nach der Wende die Ostberliner Zuschauer zunächst weniger ins Theater gingen als zu DDR-Zeiten, konnte sich in Berlin in den 1990er Jahren auf dieser Grundlage schnell wieder eine gemeinsame Theaterkultur entwickeln, in der sich in den Theatern im Stadtteil Mitte, aber auch in der Spielstätte der Freien Bühnen das Publikum schneller als andernorts mischte. Neben Abgesängen auf die DDR (etwa Heiner Müllers Hamlet-Inszenierung, in die er seine Hamletmaschine einfügte, oder Castorfs John Gabriel Borkman) waren nun Inszenierungen zu sehen, in denen es direkt um die Folgen der Wiedervereinigung ging (Schleefs Wessis in Weimar von Hochhuth am Berliner Ensemble sowie Castorfs Inszenierungen). Castorf holte dann mit Christoph Marthaler und Christoph Schlingensief eine neue Generation von

41 Ebd., S. 112.

421989 konnte das Stück Die Übergangsgesellschaft von Volker Braun (Maxim Gorki Theater/Inszenierung Thomas Langhoff) als erste DDR-Produktion gezeigt werden. 
Westregisseuren in sein Team. 1996 überträgt der Ostberliner Intendant Thomas Langhoff dem jungen Westdeutschen Thomas Ostermeier die künstlerische Leitung der Baracke des Deutschen Theaters. So bricht in der Mitte der 1990er Jahre in Berlin eine neue Theaterepoche an, in der Ost und West anders und mehr als in anderen Städten zusammenarbeiten, zusammenstoßen und zusammenfinden, in der sich neue Ästhetiken entwickeln und Berlin wieder zu einem Mittelpunkt des, freilich auch weiterhin dezentralen, Theaterlebens in den deutschsprachigen Ländern wird. Die Grundlage dazu war eine gemeinsame ästhetische Kultur, die sich trotz allem ,über die Mauer hinweg' hatte entwickeln können. Trotz der Krise eine aufregende Zeit: $\frac{(1: 2) x(1+1)}{2}=1$. Ganz im Sinne von Pippi Langstrumpf.

\section{Zusammenfassung}

Die Autorin zeichnet die Entwicklung der Berliner Theaterlandschaft von der Nachkriegszeit über Teilung und Wende bis in die Mitte der 1990er Jahre nach. Sie geht dabei vor allem der Frage nach, inwiefern es in diesem Zeitraum „ungekappte Taue“ (August Everding) zwischen Ost- und Westtheater in der geteilten Stadt gegeben hat oder ob von einer doppelten Struktur mit jeweils eigenen Bezugsystemen auszugehen ist. Dabei bezieht sie sich zunächst auf die Struktur, den Status, die Geschichte und die geographische Verteilung wichtiger Spielstätten, erklärt dann die konkurrierenden Theatersysteme und geht vor allem Fragen der politischen Einflussnahme auf das Theater im Kalten Krieg nach. Ausgehend von der Darstellung der Entwicklung nach der Wende fragt sie abschließend, auf welche biographischen und ästhetischen Kontakte die Zusammenführung der Theaterlandschaften aufbauen konnte.

\section{Résumé}

L'article retrace l'évolution du paysage théâtral à Berlin, de l'après-guerre jusque dans les années 1990, en prêtant une attention particulière aux moments charnières de la construction du Mur et de la réunification. Y a-t-il eu un „cordon non coupé “ (August Everding) entre le théâtre de l'Est et de l'Ouest ou faut-il partir de l'idée qu'il y a eu un double paysage théâtral pendant la séparation de la ville? Comment les deux moitiés du paysage théâtral se sont-elles remises ensemble en 1990? Pour répondre à ces questions, l'auteure se réfère d'abord à la structure du paysage théâtral dans Berlin Est et Ouest, situe géographiquement et historiquement les lieux les plus importants avant d'aborder les systèmes et conditions de travail, en mettant l'accent sur le rôle du politique à l'époque de la Guerre froide. Passant en revue l'évolution dans ces domaines après le „tournant" de 1990, elle demande enfin sur quels contacts biographiques et esthétiques la réunification théâtrale pouvait s'appuyer. 